\title{
Prevalence of Anaemia and Its Associated Factors among Type 2 Diabetes Mellitus Patients in University of Gondar Comprehensive Specialized Hospital
}

\author{
Sewnet Adem Kebede $\mathbb{D}^{1},{ }^{1}$ Biruk Shalmeno Tusa $\mathbb{D}^{2},{ }^{2}$ and Adisu Birhanu Weldesenbet $\mathbb{D}^{2}$ \\ ${ }^{1}$ Department of Epidemiology and Biostatistics, Institute of Public Health, College of Medicine and Health Sciences, \\ University of Gondar, Gondar, Ethiopia \\ ${ }^{2}$ Department of Epidemiology and Biostatistics, College of Health and Medical Sciences, Haramaya University, \\ Haramaya, Ethiopia \\ Correspondence should be addressed to Sewnet Adem Kebede; sewnetme1@gmail.com
}

Received 16 October 2020; Revised 1 February 2021; Accepted 4 February 2021; Published 10 February 2021

Academic Editor: Gunanidhi Dhangadamajhi

Copyright (c) 2021 Sewnet Adem Kebede et al. This is an open access article distributed under the Creative Commons Attribution License, which permits unrestricted use, distribution, and reproduction in any medium, provided the original work is properly cited.

\begin{abstract}
Background. Anaemia is one of the commonest blood disorders seen in patients with diabetes. In Ethiopia, chronic illnesses are tremendously raising with their complications. But very little research has been conducted, particularly on anaemia among diabetes mellitus (DM) patients. Therefore, this study aimed at assessing the prevalence of anaemia and associated factors among type 2 diabetes mellitus patients in Northwest Ethiopia. Methods. A cross-sectional study design was employed at University of Gondar Comprehensive Specialized Hospital from March 1 to April 15, 2019, among 372 type 2 diabetes mellitus patients (T2DM). Multivariable logistic regression analysis was fitted, and the corresponding adjusted odds ratio (AOR) and 95\% CI were used to identify factors associated with anaemia. Level of significance was declared at the $p$ value less than 0.05 . Results. The study revealed $8.06 \%$ (95\% CI: 5.68-11.31\%) of the participants were anaemic. Being male $(\mathrm{AOR}=2.74, \mathrm{CI}: 1.02,7.38)$, combined type of treatment $(\mathrm{AOR}=8.38, \mathrm{CI}: 1.66,42.25)$, having diabetes-related microvascular complications $(\mathrm{AOR}=3.24$, $\mathrm{CI}: 1.14,9.26)$, and hypertension $(\mathrm{AOR}=0.01, \mathrm{CI}: 0.002,0.06)$ were the significant factors associated with anaemia. Conclusions. The finding of the current study revealed low prevalence of anaemia among T2DM patients. Sex, type of treatment, diabetes-related microvascular complications, and hypertension were factors associated with anaemia. Assessment of haemoglobin levels among T2DM patients may help to prevent ensuing microvascular complications. Incorporate anaemia screening into the routine assessment of diabetic complication particularly for those who are hypertensive and took combined treatment to allow early appreciation and treatment of anaemia and later improve the overall care of patients with diabetes.
\end{abstract}

\section{Introduction}

Anaemia is a condition in which the number of healthy red blood cells is lower than normal in the body and/or lower than the normal amount of haemoglobin in the red blood cells [1-4]. Anaemia is an indicator of both poor nutrition and poor health [5].

Anaemia is a serious global public health problem that affects populations in both rich and poor countries. Globally, anaemia affects 1.62 billion people which correspond to $24.8 \%$ of the population [6]. It occurs at all stages of the life cycle but is more prevalent in pregnant women $(40 \%)$ and young children $(42 \%)[2,6]$.
In Ethiopia, 57\% of children aged 6-59 months suffered from some degree of anaemia. Twenty-five percent of children are classified with mild anaemia, $29 \%$ with moderate anaemia, and 3\% with severe anaemia. Twenty-four percent and fifteen percent of women and men in Ethiopia are anaemic, respectively. Eighteen percent of women are classified as mildly anaemic, 5\% moderately anaemic, and $1 \%$ severely anaemic [7].

There is considerable evidence that anaemia exacerbates severity and impairs the outcome of peripheral small vessel disease in diabetic patients [8]. Anaemia can make certain complications (diabetic neuropathy, diabetic nephropathy, and diabetic retinopathy) more likely to occur, and it can 
worsen the kidney, heart, and artery diseases, which are more common in people with diabetes $[8,9]$.

There is a significant association between haemoglobin concentration and fasting blood glucose. High incidence of anaemia is likely to occur in patients with poorly controlled diabetes and in patients with diabetes and renal insufficiency [10]. Some studies have shown that reduced erythropoietin production and anaemia happen earlier in people with diabetes and kidney disease than in those with kidney disease and no diabetes [1].

Anaemia in DM patients is a common condition, but it often goes unrecognized and not treated. Its symptoms are vague and easily mistaken for symptoms of other serious or chronic diseases. But even mild anaemia can significantly lower one's quality of life, and untreated anaemia can have serious long-term health effects $[1,11]$.

Even though several studies have been conducted in different parts of the world on anaemia among T2DM patients, its magnitude varies among their findings up to $63 \%$ in Pakistan [12], $41.4 \%$ in Cameroon, $39.4 \%$ in Malaysia, $18 \%$ in India, $55.5 \%$ in Saudi, and $34.8 \%$ in Ethiopia [13]. Such a difference will make the understanding of the exact magnitude of anaemia among T2DM patients.

In Ethiopia, chronic illnesses are tremendously raising with their complications. But very little research has been conducted, particularly on anaemia among DM patients, although it has been reported as the common complication of DM. Thus, knowing its current magnitude is very important for policy makers to design an intervention and strengthen regular screening and management of anaemia among DM patients and can be used as evidence for early sign of kidney problem and other diabetes-related complications and improving DM patient's health-related quality of life through prevention of diabetes-related complications. Hence, this research aimed to determine the prevalence of anaemia and its associated factors among T2DM patients at University of Gondar Comprehensive Specialized Hospital (UGCSH) in Northwest Ethiopia.

\section{Methods and Materials}

2.1. Study Design, Setting, and Population. A cross-sectional study design was employed at UGCSH from March 1 to April 15, 2019. UGCSH is found in Gondar town of Amhara regional state, which is located $743 \mathrm{~km}$ northwest of the capital Addis Ababa, Ethiopia, and it serves Gondar and surrounding zones. Type $2 \mathrm{DM}$ patients attending the outpatient's clinic of the hospital for routine follow-up during the study period were included. All patients with a known hematologic disease, those who received a blood transfusion in the preceding 3 months, and those who were pregnant women were excluded.

2.2. Sample Size Determination. The required sample size of the study was determined using single population proportion formula by considering $34.8 \%$ prevalence rate of anaemia based on a previous study in people with diabetes in Ethiopia [13], $Z=$ the level of statistical significance with a
$95 \%$ confidence interval (CI) of 1.96, and precision level of 0.05 . Then, the minimum sample size obtained was 348 . After adding $10 \%$ to account for nonrespondents, a total of 382 diabetic patients were included in the study. A systematic random sampling technique (i.e., every two patients) was employed to select the study participants.

2.3. Data Collection. Data were collected by using a structured data extraction checklist. Patient intake form, followup card, and DM registration book were used as data sources. Sociodemographic characteristics, baseline, and follow-up clinical and laboratory data were collected from patient cards. Four data collectors and one supervisor who are health professionals were recruited. Two-day training was given for the data collectors and supervisor on how to retrieve records as per data extraction sheet.

2.4. Operational Definitions. Patients were classified as anaemic according to the World Health Organization (WHO) criteria $(\mathrm{Hg}<12 \mathrm{~g} / \mathrm{dl}$ for females and $<13 \mathrm{~g} / \mathrm{dl}$ for males) [3]. Microvascular complications of diabetes are those long-term complications that affect small blood vessels. These typically include retinopathy, nephropathy, and neuropathy. Macrovascular complications of diabetes are primarily diseases of the coronary arteries, peripheral arteries, and cerebrovasculature [14]. For good glycaemic control, an average of four consecutive fasting blood glucose measurement was $\leq 130 \mathrm{mg} / \mathrm{dl}$, and for poor glycaemic control, an average of four consecutive fasting blood glucose measurement was $>130 \mathrm{mg} / \mathrm{dl}$ [15].

2.5. Statistical Analysis. The data were checked for inconsistencies, coding error, completeness, clarity, and missing values before they were entered. The data were entered using Epi-info 7 and exported to STATA 14.1 statistical software for further data cleaning and statistical analysis. Descriptive statistical analysis such as frequency, percentage, cross tabulation, mean, and standard deviation were performed. Multivariable logistic regression analysis was fitted, and the corresponding adjusted odds ratio (AOR) and 95\% CI were used to identify factors associated with anaemia. A $p$ value $<0.05$ was used to characterize statistically significant results.

\section{Results}

3.1. Characteristics of Study Participants. A total of 372 T2DM patients, of which $230(61.83 \%)$ were females, were included in the study. More than half of the participants (206 (55.38)) were aged above 60 years. From the total number of the participants, 138 (37.10\%) had at least one of diabetesrelated microvascular complications, whereas fifty-six of them $(15.05 \%)$ had at least one diabetes-related macrovascular complications. The duration of DM ranged from 2 up to 19 years, with a mean $( \pm \mathrm{SD})$ of $8.87 \pm 3.69$ years. One hundred (26.88\%) participants were hypertensive, with $76(20.43 \%)$ and $45(12.10 \%)$ participants having SBP of $>140 \mathrm{mmHg}$ and DBP 
of $>90 \mathrm{mmHg}$, respectively. The average of four consecutive fasting blood sugar levels (FBS) during study periods is with a mean $( \pm$ SD) of $204.50 \pm 57.10 \mathrm{mg} / \mathrm{dl}$ (Table 1).

3.2. Prevalence of Anaemia among T2DM Patients. The overall prevalence of anaemia in the study participants was found to be $8.06 \%$ (95\% CI: $5.68-11.31 \%$ ); $10.56 \%$ of diabetic males and $6.52 \%$ of diabetic females were found to be anaemic. Of these 372 patients, $14.49 \%$ patients with diabetes-related microvascular complications and $17.86 \%$ patients with diabetes-related macrovascular complications had anaemia.

3.3. Factors Associated with Anaemia among T2DM Patients. Table 2 shows the factors associated with anaemia among T2DM patients. Sex, type of treatment, diabetes-related microvascular complications, and hypertension were the significant factors associated with anaemia. Diabetic male patients were 2.74 times more likely to be anaemic than diabetic female patients. The odds of developing anaemia in patients with at least one diabetes-related microvascular complication were 3.24 times more likely as compared with those without any microvascular complications. The study also showed that the odds of developing anaemia for patients who take combined treatment were 8.38 times higher than those patients who took oral glycaemic agent only. In addition, there was a statistically significant relationship between hypertension and anaemia.

\section{Discussion}

The current study demonstrated the prevalence of anaemia among T2DM patients in UOGCSH to be $8.06 \%$. This number is lower than the finding of a hospital-based crosssectional study performed in Harari region, Eastern Ethiopia (34.8\%), and northeast of the country $(20.1 \%)[13,16]$; this prevalence is also lower than previous studies in Iran (30.4\%), India (18\%), and Malaysia (39.4\%) [17-19]. Such variations in the magnitude of anaemia among diabetic patients might be due to differences in the cutoff value used to measure it, sample size, and variations in the overall characteristics of the study area that could be related to the prevalence of anaemia among T2DM patients.

The finding of the current study showed that gender was significantly associated with anaemia. Anaemia is more likely to occur in male patients compared to female patients. Similar finding have been reported in Ethiopia [13]. However, this finding was contraindicating with another study performed in Pakistan [12]. The possible explanation could relate to differences involving genetic factors and number of female patients who are menopause because of the effect of menstruation on iron stores.

In this study, diabetes-related microvascular complication is significantly associated with the occurrence of anaemia. Consistent with the present finding, the increased ratio for developing anaemia has also been found in the previous study conducted in China [20], Egypt [9], Cameroon [21], and Iran [17]. This could be explained as
TABLE 1: Sociodemographic and clinical characteristics of T2DM patients at UGCSH, Northwest Ethiopia, $2019(n=372)$.

\begin{tabular}{|c|c|c|}
\hline Variables & $\begin{array}{l}\text { Frequency } \\
(n=372)\end{array}$ & Percentage (\%) \\
\hline \multicolumn{3}{|l|}{ Sex } \\
\hline Male & 142 & 38.17 \\
\hline Female & 230 & 61.83 \\
\hline \multicolumn{3}{|l|}{ Age (years) } \\
\hline$\leq 60$ & 166 & 44.62 \\
\hline$>60$ & 206 & 55.38 \\
\hline \multicolumn{3}{|l|}{ Duration of DM } \\
\hline$\leq 5$ & 65 & 17.47 \\
\hline $6-10$ & 193 & 51.88 \\
\hline$>10$ & 114 & 30.65 \\
\hline \multicolumn{3}{|l|}{ Hypertension } \\
\hline Yes & 100 & 26.88 \\
\hline No & 272 & 73.12 \\
\hline \multicolumn{3}{|l|}{ SBP } \\
\hline$\leq 140$ & 296 & 79.57 \\
\hline$>140$ & 76 & 20.43 \\
\hline \multicolumn{3}{|l|}{ DBP } \\
\hline$\leq 90$ & 327 & 87.90 \\
\hline$>90$ & 45 & 12.10 \\
\hline \multirow{2}{*}{\multicolumn{3}{|c|}{$\begin{array}{l}\text { Microvascular } \\
\text { complication }\end{array}$}} \\
\hline & & \\
\hline No & 234 & 62.90 \\
\hline Yes & 138 & 37.10 \\
\hline \multicolumn{3}{|l|}{$\begin{array}{l}\text { Macrovascular } \\
\text { complication }\end{array}$} \\
\hline No & 316 & 84.95 \\
\hline Yes & 56 & 15.05 \\
\hline \multicolumn{3}{|l|}{ Glycaemic control } \\
\hline Poor & 346 & 93.01 \\
\hline Good & 26 & 6.99 \\
\hline \multicolumn{3}{|l|}{ Type of treatment } \\
\hline $\begin{array}{l}\text { Oral hypoglycaemic } \\
\text { agent }\end{array}$ & 329 & 88.44 \\
\hline Combined & 43 & 11.56 \\
\hline
\end{tabular}

DBP, diastolic blood pressure; SBP, systolic blood pressure. Combined both insulin and oral hypoglycaemic agents.

anaemia is associated with a reduction in both the number of red blood cells and antioxidant potential of erythrocytes, which may lead to characteristics diabetic complications [22]. In addition, some studies reported that anaemia may modulate the activity of molecular signaling pathways that lead to progressive organ damage [23]. The odds of anaemia were higher among patients who were taking combined treatment compared to those who were taking oral hypoglycaemic agents. The possible explanation could be due to malabsorption of vitamin B12 and the destruction of red blood cell due to the adverse effect of the drugs and also the synergetic effect of the two drugs.

In this study, we found that hypertension was the strongest risk factor for anaemia among T2DM patients. The main cause could be, in diabetic patients, the risk of renal impairment, thus increasing the subsequent development of anaemia. In addition, nutritional deficiencies especially iron deficiency and chronic inflammation can be the cause [24]. This finding is consistent with a cross-sectional study performed in Ethiopia [25]. The study has its limitation as this 
TABLE 2: Multivariable logistic regression of variables associated with anaemia among T2DM patients in UGCSH, Northwest Ethiopia, 2019.

\begin{tabular}{|c|c|c|c|c|}
\hline \multirow{2}{*}{ Variables } & \multicolumn{2}{|c|}{ Anaemia } & \multirow{2}{*}{ COR $(95 \% \mathrm{CI})$} & \multirow{2}{*}{ AOR $(95 \% \mathrm{CI})$} \\
\hline & Yes & No & & \\
\hline \multicolumn{5}{|l|}{ Sex } \\
\hline Female & 15 & 215 & 1 & 1 \\
\hline Male & 15 & 127 & $1.69(1.08,3.58)$ & $2.74(1.02,7.38)^{*}$ \\
\hline \multicolumn{5}{|l|}{ Age } \\
\hline$\leq 60$ & 12 & 154 & 1 & 1 \\
\hline$>60$ & 18 & 188 & $1.23(0.57,2.63)$ & $0.60(0.22,1.64)$ \\
\hline \multicolumn{5}{|l|}{ SBP } \\
\hline$\leq 140$ & 25 & 271 & 1 & 1 \\
\hline$>140$ & 5 & 71 & $0.57(0.19,1.71)$ & $0.66(0.15,2.95)$ \\
\hline \multicolumn{5}{|l|}{ DBP } \\
\hline$\leq 90$ & 24 & 303 & 1 & 1 \\
\hline$>90$ & 6 & 39 & $0.49(0.11,2.16)$ & $0.22(0.03,1.51)$ \\
\hline \multicolumn{5}{|l|}{ Duration of DM } \\
\hline$\leq 5$ years & 5 & 60 & 1 & 1 \\
\hline $6-10$ years & 18 & 175 & $1.44(1.27,7.88)$ & $1.45(0.26,8.24)$ \\
\hline$>10$ years & 7 & 107 & $1.55(0.39,6.09)$ & $0.54(0.09,3.33)$ \\
\hline \multicolumn{5}{|l|}{ Type of treatment } \\
\hline Oral hypoglycaemic agent & 25 & 304 & & 1 \\
\hline Combined & 5 & 38 & $1.68(1.25,4.43)$ & $8.38(1.66,42.25)^{* *}$ \\
\hline \multicolumn{5}{|l|}{ Microvascular complications } \\
\hline No & 10 & 224 & 1 & 1 \\
\hline Yes & 20 & 118 & $3.79(1.72,8.37)$ & $3.24(1.14,9.26)^{*}$ \\
\hline \multicolumn{5}{|l|}{ Macrovascular complications } \\
\hline No & 20 & 296 & 1 & 1 \\
\hline Yes & 10 & 46 & $3.21(1.42,7.31)$ & $1.15(0.36,3.63)$ \\
\hline \multicolumn{5}{|l|}{ Glycaemic control } \\
\hline Good & 5 & 21 & 1 & 1 \\
\hline Poor & 25 & 321 & $0.45(0.14,1.39)$ & $0.24(0.05,1.21)$ \\
\hline \multicolumn{5}{|l|}{ Hypertension } \\
\hline Yes & 6 & 266 & 1 & 1 \\
\hline No & 24 & 76 & $0.03(0.008,0.10)$ & $0.01(0.002,0.06)^{* * *}$ \\
\hline
\end{tabular}

${ }^{* * *} p$ value $<0.001 ;{ }^{* *} p$ value $<0.01 ;{ }^{*} p$ value $<0.05$. CI, confidence interval; DBP, diastolic blood pressure; SBP, systolic blood pressure; AOR, adjusted odds ratio; COR, crude odds ratio.

study was conducted based on secondary data, data on some potentially important predictors which help to know that the dietary pattern of the patient was not assessed. Moreover, since this study used a cross-sectional study design, we cannot report the cause and effect relationship of microvascular complications and anaemia.

\section{Conclusion}

The finding of the current study revealed low prevalence of anaemia among T2DM patients. Sex, type of treatment, diabetes-related microvascular complications, and hypertension were factors associated with anaemia. Assessment of haemoglobin levels among T2DM patients may help to prevent ensuing diabetes-related microvascular complications. Incorporate anaemia screening into the routine assessment of diabetic complication particularly for those who are hypertensive and took combined treatment to allow early appreciation and treatment of anaemia and later improve the overall care of patients with diabetes.

\section{Abbreviations}

AOR: Adjusted odds ratio
COR: Crude odds ratio

DBP: Diastolic blood pressure

DM: Diabetes mellitus

FBS: $\quad$ Fasting blood sugar

HR: Hazard ratio

HTN: Hypertension

SBP: $\quad$ Systolic blood pressure

T2DM: Type 2 diabetes mellitus.

\section{Data Availability}

The data used to support the findings of this study are included within this article.

\section{Ethical Approval}

Before the commencement of the study, ethical clearance was obtained from the Institutional Review Board of the University of Gondar. Then, permission letters from officials of University of Gondar Comprehensive Specialized Hospital, Department of Internal Medicine, were processed before data collection. To ensure confidentiality, patient 
names were not included; instead, code numbers were assigned to depict the results.

\section{Conflicts of Interest}

The authors declare that they have no conflicts of interest.

\section{Authors' Contributions}

Conception of the work, design of the work, acquisition of data, and analysis and interpretation of data were done by SA. Data curation, drafting the article, revising it critically for intellectual content, validation, and final approval of the version to be published were done by SA, BS, and AB. All authors read and approved the final manuscript.

\section{Acknowledgments}

The authors express their appreciation to University of Gondar Comprehensive Specialized Hospital, particularly the DM clinic staff for their kind cooperation during data collection. The authors are also grateful to the data collectors.

\section{References}

[1] Diabetes Self Management, Anemia, https://www. diabetesselfmanagement.com/managing-diabetes/generalhealth-issues/anemia/, 2020.

[2] WHO, Anaemia, World Health Organization, Geneva, Switzerland, 2020, https://www.who.int/health-topics/ anaemia\#tab=tab_1.

[3] WHO, Haemoglobin Concentrations for the Diagnosis of Anaemia and Assessment of Severity, World Health Organization, Geneva, Switzerland, 2011.

[4] W. Targets, 2025: Anaemia Policy Brief, World Health Organization, Geneva, Switzerland, 2014.

[5] WHO, Focusing on Anaemia: Towards an Integrated Approach for Effective Anaemia Control: Joint Statement by the World Health Organization and the United Nations Children's Fund, World Health Organization, Geneva, Switzerland, 2004.

[6] B. De Benoist, M. Cogswell, I. Egli, and E. McLean, Worldwide Prevalence of Anaemia 1993-2005, WHO Global Database of Anaemia, Geneva, Switzerland, 2008.

[7] C. ICF, Ethiopia Demographic and Health Survey 2016, CSA and ICF, Addis Ababa, Ethiopia, 2016.

[8] WebMD, Diabetes and Anemia, WebMD, New York, NY, USA, 2020, https://www.webmd.com/diabetes/diabetes-andanemia.

[9] M. Nasrat, M. Y. Samar, N. E. Esheba, and H. E. Mohammed, "The relation between anemia and microvascular complications in patients with type 2 diabetes mellitus," The Medical Journal of Cairo University, vol. 86, pp. 947-954, 2018.

[10] S. Antwi-Bafour, S. Hammond, J. K. Adjei, R. Kyeremeh, A. Martin-Odoom, and I. Ekem, "A case-control study of prevalence of anemia among patients with type 2 diabetes," Journal of Medical Case Reports, vol. 10, no. 1, pp. 1-8, 2016.

[11] M. C. Thomas, "Anemia in diabetes: marker or mediator of microvascular disease?," Nature Clinical Practice Nephrology, vol. 3, no. 1, pp. 20-30, 2007.

[12] A. Sharif, S. Younus, K. Baig, and N. H. Ali, "Prevalence and risk of anemia in type-2 diabetic patients," Health, vol. 6, no. 12, pp. 1415-1419, 2014.
[13] A. Bekele, K. Teji Roba, G. Egata, and B. Gebremichael, "Anemia and associated factors among type-2 diabetes mellitus patients attending public hospitals in Harari Region, Eastern Ethiopia," PLoS One, vol. 14, no. 12, Article ID e0225725, 2019.

[14] R. S. Zimmerman, Diabetes Mellitus: Management of Microvascular and Macrovascular Complications, Cleveland Clinic: Centers for Continuing Education, Lyndhurst, $\mathrm{OH}$, USA, 2016.

[15] T. Kassahun, T. Eshetie, and H. Gesesew, "Factors associated with glycemic control among adult patients with type 2 diabetes mellitus: a cross-sectional survey in Ethiopia," $B M C$ Research Notes, vol. 9, no. 1, p. 78, 2016.

[16] M. M. Taderegew, T. Gebremariam, A. A. Tareke, and G. G. Woldeamanuel, "Anemia and its associated factors among type 2 diabetes mellitus patients attending debre berhan referral hospital, north-east Ethiopia: a cross-sectional study," Journal of Blood Medicine, vol. 11, p. 47, 2020.

[17] M. S. Hosseini, Z. Rostami, A. Saadat, S. M. Saadatmand, and E. Naeimi, "Anemia and microvascular complications in patients with type 2 diabetes mellitus," Nephro-Urology Monthly, vol. 6, no. 4, 2014.

[18] S. C. Thambiah, I. N. Samsudin, E. George, L. K. Ranjit, N. S. Saat, and Z. Hussein, "Anaemia in type 2 diabetes mellitus (T2DM) patients in Hospital Putrajaya," Malaysian Journal of Medicine and Health Sciences, vol. 11, no. 1, pp. 49-61, 2015.

[19] G. B. Rathod, P. Parmar, S. Rathod, and A. Parikh, "Prevalence of anemia in patients with type 2 diabetes mellitus at Gandhinagar, Gujarat, India," International Archives of Integrated Medicine, vol. 3, no. 3, pp. 12-16, 2016.

[20] B. B. He, M. Xu, L. Wei et al., "Relationship between anemia and chronic complications in Chinese patients with type 2 diabetes mellitus," Archives of Iranian Medicine, vol. 18, no. 5, pp. 277-283, 2015.

[21] V. F. Feteh, S.-P. Choukem, A.-P. Kengne, D. N. Nebongo, and M. Ngowe-Ngowe, "Anemia in type 2 diabetic patients and correlation with kidney function in a tertiary care subSaharan African hospital: a cross-sectional study," $B M C$ Nephrology, vol. 17, no. 1, p. 29, 2016.

[22] A. Klemm, C. Voigt, M. Friedrich et al., "Determination of erythrocyte antioxidant capacity in haemodialysis patients using electron paramagnetic resonance," Nephrology Dialysis Transplantation, vol. 16, no. 11, pp. 2166-2171, 2001.

[23] R. Deicher and W. H. Hörl, "Anaemia as a risk factor for the progression of chronic kidney disease," Current Opinion in Nephrology and Hypertension, vol. 12, no. 2, pp. 139-143, 2003.

[24] R. M. O. Ximenes, A. C. P. Barretto, and E. Silva, "Anemia in heart failure patients: development risk factors," Revista Brasileira de Cardiologia, vol. 27, no. 3, pp. 189-194, 2014.

[25] T. Fiseha, A. Adamu, M. Tesfaye, and A. Gebreweld, "Prevalence of anemia in diabetic adult outpatients in Northeast Ethiopia," PLoS One, vol. 14, no. 9, Article ID e0222111, 2019. 